\title{
A REVIEW ON GENERAL SELF-ORGANIZED TREE-BASED ENERGY- BALANCE ROUTING PROTOCOL FOR WIRELESS SENSOR NETWORK
}

\author{
Shikha Chhabra (1), Vishal Arora (2) \\ (1) Research Scholar, Department of Computer Science Engineering, SBSSTC, Ferozepur \\ shikha61188@gmail.com \\ (2) Assistant Professor, Department of Computer Science Engineering, SBSSTC, Ferozepur \\ vishal.fzr@gmail.com
}

\begin{abstract}
The quick escalations in network multimedia devices have permitted extra concurrent digital services: video conferencing, online playoffs as well as remote learning to nurture for conform e-net jobs. WSNs have become major area of research in computational theory due to its wide-ranging applications. But due to limited battery power the energy expenditure has become key drawback of WSNs protocols. Although several protocols has been proposed so far to improve the energy efficiency more however still a lot enhancement can be done. GSTEB has shown fairly significant results over the on hand WSN protocols. The general purpose of this work is to study the architecture of WSN and the problems associated with it. At the end of this paper appropriate future guidelines are given to further improve this work.
\end{abstract}

\section{Keywords}

Wireless Sensor Networks, Protocol, Cluster Head, Tree, GSTEB, WSN.

\section{INTRODUCTION}

A wireless sensor network is a technology that emerges as a consequence of the evolution of network technology along with microelectronics and micromechanical devices. It is a new concept, a view towards the future, a clear consequence of the new steps forward in the communications field. In few words a wireless sensor network, is a network that could contain from a couple to many small nodes with sensors attached and communications capabilities to transmit and receive information. The data acquired by these sensors is then transmitted along a network from one node to another until it is collected in a central unit, normally connected to a PC. If the original expectations created around this new network concept come true, it will improve monitoring and control systems used nowadays in the environment, medical, industry, consumer, and military sectors. With the help of this technology such systems will now be capable of raising a huge amount of data and results which will be available for analysis in real time. This could lead to a new era of monitoring and controlling processes as they are occurring, something that it was not possible before in some cases without the intervention of persons and complex equipment. Another advantage in the use of wireless technology is the reduction in cost that cabling deployment has in current systems and also the possibility of performing measurements in inaccessible places. In ad-hoc network each and every nodes are allow to communicate with each other without any fixed infrastructure. This is actually one of the features that differentiate between ad-hoc and other wireless technology like cellular networks and wireless LAN which actually required infrastructure based communication like through some base station. [2]. Wireless sensor network are one of the category belongs to ad-hoc networks. Sensor network are also composed of nodes. Here actually the node has a specific name that is "Sensor" because these nodes are equipped with smart sensors [2]. A sensor node is a device that converts a sensed characteristic like temperature, vibrations, pressure into a form recognize by the users. Wireless sensor networks nodes are less mobile than ad-hoc networks. So mobility in case of ad-hoc is more. In wireless sensor network data are requested depending upon certain physical quantity. So wireless sensor network is data centric. A sensor consists of a transducer, an embedded processor, small memory unit and a wireless transceiver and all these devices run on the power supplied by an attached battery [1].

Wireless Sensor Network (WSN) is a class of wireless adhoc networks which consists of spatially distributed autonomous sensor nodes to monitor physical or environmental conditions, such as temperature, sound, pressure, etc. at different locations. Energy consumption is the core issue in wireless sensor networks because nodes are battery operated. It is desirable to make these nodes as cheap and energy-efficient as possible and rely on their large numbers to obtain high quality results. Consequently many protocols have been proposed in order to minimize the energy consumption of these nodes. Wireless Sensor Network (WSN) is a type of wireless Ad-Hoc network in which large numbers of sensor nodes are deployed in the application field [1]. Sensor nodes are inexpensive and low power devices. Each node consists of four main units: Sensing Unit, Processing Unit, Communicating Unit and Power Unit. In sensing unit one or more sensors are placed to sense different environment parameters like sound, temperature, vibration, pressure, motion and etc., based on application requirement. Sensed data's (analog or digital) are processed and aggregated in the processing unit.

The need for energy-efficient infrastructures for sensor networks is becoming increasingly important. Wireless sensor networks are networks consisting of many sensor nodes that communicate over a wireless media. A sensor node is equipped with a sensor module, a processor, a radio module and a battery. Since the battery limits the lifetime of the sensor nodes it also limits the lifetime of the sensor network, thus energy efficiency is a major issue for sensor networks. An important goal in many sensor networks is to monitor an area as long time as possible. Hence, it is important to distribute energy consumption evenly across the network. When the energy consumption is evenly distributed, the major part of the sensor nodes will stay alive approximately equally long time. This enables continued information gathering throughout the whole network area during the lifetime of the network. 


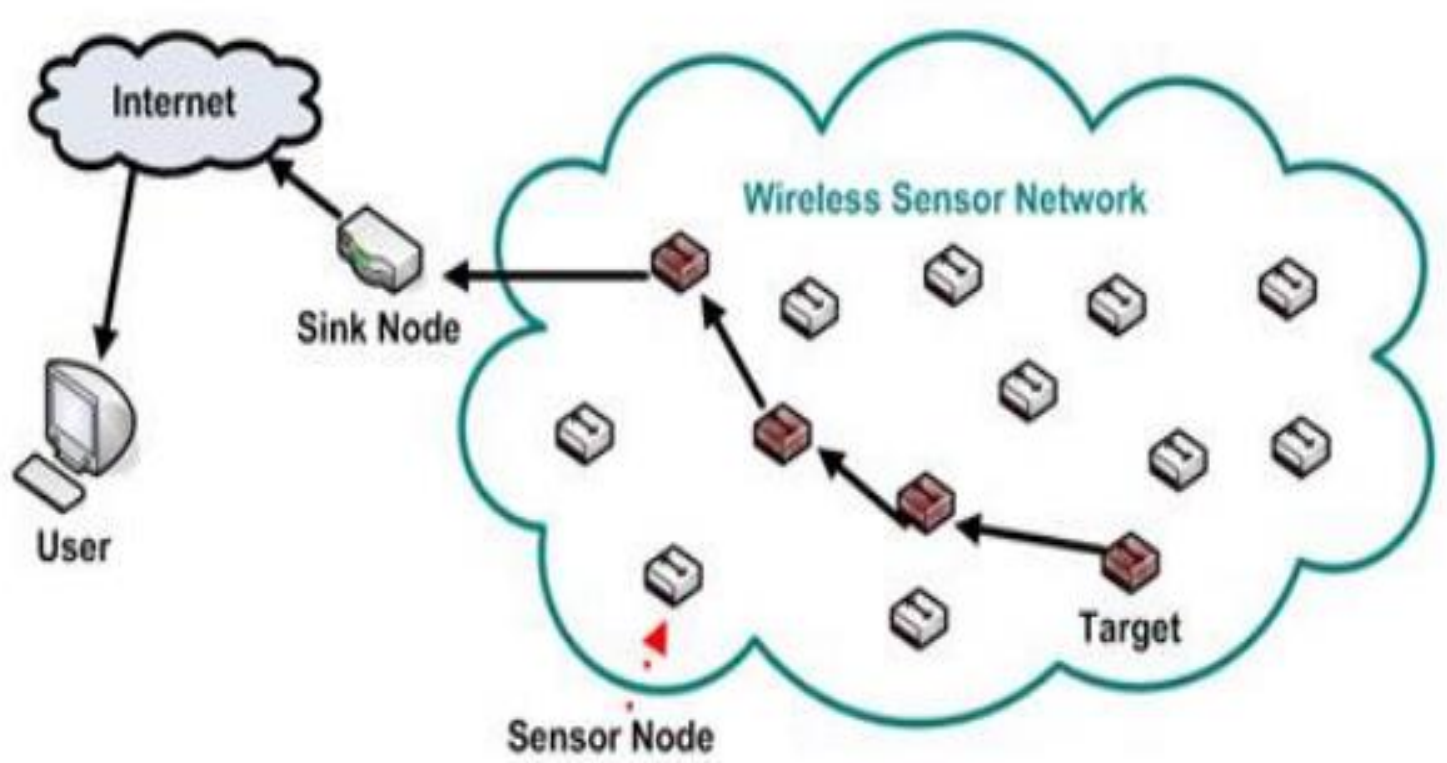

Figure 1. Wireless Sensor Network

The most power-consuming activity of a sensor node is typically radio communication [10]. Hence, radio communication must be kept to an absolute minimum. Some sensor nodes become cluster heads and collect all traffic from their respective cluster. The cluster head aggregates the collected data and then sends it to its base station. When using clustering, the workload on the cluster head is thus larger than for non-cluster heads. The cluster heads should therefore be changed several times during the lifetime of the sensor network in order to distribute the extra workload and energy consumption evenly. Sensor nodes are deployed for pervasive computing. Each of the sensor node is capable of limited amount of processing, upon coordination with the other node's information, gains the power to accomplish complex functionalities. Thus in Wireless Sensor Network (WSN), a collection of the sensor coordinates well with each other, using wireless communication link, in order to pursue some specific tasks.

\section{CHARACTERISTIC OF WIRELESS SENSOR NETWORK}

- Infrastructure less: In wireless sensor network every node that is equipped with smart sensor which are communicate with each other without any fixed infrastructure. So communication overhead will be less.

- Mobility: Mobility of nodes in wireless sensor networks is less. Due to this kind of feature they are used in event tracking purposes like motion detection of video frames etc.

- Multi-Hoping: Wireless sensor Networks composed of several nodes and they are communicating with each other and describe several paths to several node. Here actually the packet traverses from one node to another node to reach the destination through several paths. Due to this Multi-hop features energy associated with each node can be conserved [4].

- Openness: Wireless sensor network access information and services regardless of Geographic position.

- Network size: In wireless sensor network the node are scattered in large geographical area.

- Homogeneous Network: A Wireless sensor network composed of homogeneous devices that is node has same features.

- Data centric: In wireless sensor network data is requested depending upon certain physical quantity so wireless sensor network is data centric [1].

- Addressing scheme: In sensor networks addresses contain attribute-value pair .This pair of value specifies certain physical parameters to be sensed [1].

- Location Awareness: Every node should know their position relative to other node by knowing their actual location. Data are collected depending upon the location [1].

- Reliable transmission of Data: To have a proper request-response model the node should request to a proper node .This node should process the request efficiently and reply as soon as possible. So that the mechanism should be reliable.

- Fault tolerant: In wireless sensors, if one node fails then it does not affect the network operation because there are other adjacent nodes collecting similar data. The accuracy of data collected is reduced.

\section{CHALLENGES IN WIRELESS SENSOR NETWORK}

Challenges in wireless sensor network arise in implementation of several services. There are so many controllable and uncontrollable parameter [4] by which the implementation of wireless sensor network affected such as. 
- Energy conservation: In wireless sensor network every node is equipped with sensor and the sensor devices are in working condition depending upon the power supplied by attached battery. To have better performance the network should operate for large time. As we know that the sensor node has small size due this small size the battery has low capacity and the available energy is very less. And in that situation the refilling or replacing of battery is impossible. It is a costly attempt. In order to avoid this problem some more energy efficient protocol are design so that the sensor node communicate efficiently by increasing both throughput and network capacity.

- Operation in antagonistic environment: Sensor network can be operating in antagonistic environmental condition. So design issues of sensor node are carefully considered. Protocol for the sensor network should be robust one. It silent about any fault occurs in system.

- Communication quality: Sensor network have very low quality communication depending upon different situation like when it operated in some unpleasant environment then communication quality is very poor. It is environment specific.

- Availability of resources: When the resources required by sensor network are unavailable then the sensor networks try hard to provide the desired QoS.

- Data processing: Data collected by many sensors may contain redundant data. So data aggregation required in network processing so that redundant data can't be transmitted number of time. It will help to conserve some amount of energy for further transmission.

- Scalability: Wireless sensor node are composed of large number of sensor node and many more nodes can be added in design stage [2].

\section{APPLICATIONS IN WIRELESS SENSOR NETWORK}

- Intrusion Detection: Due to presence of less mobility and stationary property of wireless sensor network can be used to track object event. It can be used for security purposes that are for surveillance purposes. Generally high resolution camera is equipped with sensors that can be used to form a network that monitor a restricted area access. If any outsider enter into this region without any proper authentication then some sort of signaling event occur it may be an alarm message quickly propagate to a handling authority [2].

- Avalanche prediction: Movement of large snow masses can be predicted by device equipped with sensor. GPS one of the detecting devices use for this purposes [2].

- Environment Observation and Forecasting System: The Environment Observation and Forecasting System (EOFS) is a distributed system that spans large geographic areas and monitors, models and forecasts physical processes such as environmental pollution, flooding, among others. It consists of three components: sensor stations, a distribution network, and a centralized processing farm.

\section{LITERATURE SURVEY}

Yu Xiuwu et al (2016) proposes a routing protocol that is based on monitoring area partition clustering for energy-balanced (EBPC). They divided monitoring area into several virtual partitions, controlled the number of common nodes and clusters by data fusion rate, and forwarded cluster heads data to the next partitions with minimum path selection coefficient. Samaresh Bera et al (2016) proposes a software-defined wireless sensor network architecture (Soft-WSN)-an effort to support application-aware service provisioning in Internet of Things (IOT). Detailed architecture of the proposed system is presented involving the application, control, and infrastructure layers to enable software-defined networking (SDN) in loT. Alduais et al (2016) specifies that WSN has become an enabler technology for the IOT applications which extend the physical reach of the monitoring capability. WSN as it is, possess several constraints such as limited energy availability, low memory size, and low processing speed, which are the principal obstacles to designing efficient management protocols for WSNs, what more if it concerns WSN-IOT integration. Omkar Singh et al (2016) compares three well accepted WSN routing protocols namely LEACH, FAIR and SEP for their energy pattern in heterogeneous scenario. Heterogeneity is introduced in terms of initial random energy values given to the sensor nodes. All simulations are done in MATLAB. Different parameters are used for checking the efficacy of the considered routing protocol for $\mathrm{H}$-WSN. Rani Kumari et al (2016) analyzes the performance of the various routing protocols of the wireless network in the WBAN and WSN. Moreover, the paper also compares their performance in the same network and in different network using different parameters i.e. PDR, latency and throughput etc. The research signifies that the wireless protocols needs updating to perform well in the WBAN. Hector Kaschel et al (2016) describes the mechanisms to achieve energy efficiency in a WSN, focusing particularly on routing protocols. Based on the state of the art, is presented a complete taxonomy of routing protocols used in WSN exposing their level of energy efficiency. Asdianur et al (2016) studied effectiveness in using Compressive Sensing (CS) algorithm in order to reduce measuring in IEEE 802.15.4 Standard Wireless Sensor Network (WSN). As well known, in common WSN work system, Base Station (BS) gather some information from available nodes, which the process itself consumes a lot of energy from each node. Mohd Zaki Shahabuddin et al (2016) proposes fundamental modelling of topology control algorithm to conserve individual WSN node's energy, and at the same time preserving the graph connectivity. The proposed topology control algorithm consists of three phases: 1 . Identifying connecting nodes at maximum transmission, 2. pairing nodes with shortest algorithm/minimum energy level, 3 . Calculating/setting minimum power transmission per-node for energy conservation. The algorithm works-out locally and dispenses full graph connectivity, and theoretically would be able to reduce WSN control overhead. Abhaykumar et al (2016) presents a novel scheme to improve the lifetime of a sensor node by optimizing the size of the packet such that there is no loss of data along with providing an increased lifetime for the nodes. Wireless sensor network is a rapid 
developing area with diverse applications. Smartness and interoperability of network keeps it in high demand and hence comes need for the efficiency of the system. The most important limitation on sensor node is the low power consumption. Sensor nodes carry inadequate, generally irreplaceable power sources. Therefore, while traditional networks aim to achieve high quality of service (QoS), wireless sensor network protocols must emphasis predominantly on power conservation. B. Bengherbia et al (2016) states that the wireless sensor network (WSN) represent fertile area for research and exploration, especially with enormous development in modern electronic systems, where it becomes an alternative to traditional wired systems. The wireless stems are less energy-consumptive and inexpensive, in addition it helps to reduce the complexity of wiring. Wireless sensor networks require the design of high density computing, an energy-efficient and a flexible node architecture. Gagandeep Kaur et al (2016) decreases the passive attack on sink node by decreasing the traffic on sink node. The simulation results demonstrate the proposed method can each node will compress their data before sending to cluster head. After compressing, the packet size of node will decrease. This will decrease the traffic overload. Mei Wu et al (2016) proposes a new model to efficiently control cluster-heads using heterogeneous sensors in real-time. It also proposes a generic software framework to feasibly and efficiently meet the needs of the users and applications (e.g., reliability, energy efficiency). The proposed model and its method have been implemented and evaluated in MATLAB, comparing its performance with known protocols for WSNs. The proposed model is suitable for common use because it is superior to other methods by energy efficiency. Sanaa. $S$ et al (2016) introduces an authentication protocol and simple key distributed scheme between sensor nodes. Node mobility has been taken into consideration and the work proposes a re-authentication protocol that is very efficient than the initial protocol. A wireless sensor network (WSN) consists of thousands of sensors and one base station. Sensors are deployed in the network to monitor target area and sense information according to the applied application then send this information to the base station. Roman Lara-Cueva et al (2016) presents a study in order to identify the value range of the main parameters within Carrier Sense Multiple Access (CSMA) defined in IEEE 802.15.4 that guarantee a satisfactory Wireless Sensor Networks (WSN) performance for a possible volcano monitoring application. Moreover, this study performs the comparison between test-bed in outdoor scenarios with the purpose of distinguishing the optimal number of nodes or each gateway according the main constrains given by an existing sensor network for real-time (RT) volcano monitoring system such as sampling time, packet loss, and delay.

\section{DATA AGGREGATION}

Data aggregation is as a necessary standard for wireless route selection in sensor networks. The initiative is to merge the data impending from unlike resources, remove superfluously, diminish the numerous broadcastings and hence keep the power. Data aggregation merge dissimilar data from unlike nodes by the functions like min (), max (), average (), and containment. As the network power mostly scatters on data broadcast, the power keeping algorithm has to lessen the quantity of spread data. Data aggregation is one of the effectual power keeping system that merges the data gathered via the unlike sensors by passing through a filter in which the superfluous data could lessen the quantity of spread data. In other words, data aggregation is able to improve the data exactness of network. Following are the several data aggregation techniques.

\section{SDAOC}

For WSNs, highly Secured Data Aggregation method using tree structured Orthogonal Codes (SDAOC). With tree based data aggregation shown in fig. 2, the imitation is completed in a situation among evenly supply of nodes. According to Walsh Hadamards [1] algorithm, to make twenty chip codes, selects the least power of 2. Remind that this digit ought to be larger than twenty. Hence produces 32 bit chip code, each one of them has 32 bits, except barely 20 bits of those bits are utilized. This standardized and homogenous arrangement inflicts a reduced amount of intricacy in scheming sensor networks. Consequently, the surroundings find extra anxious the projected process have further rewards than any other processes. The nodes and the broadcasting ends that can be generated among the diverse sensor nodes then the graphs made by sensors and their relationship with graph cost of the edge signify the detachment among the nodes. When graph crafting is done among the nodes, create a straddling tree above the graph. The motive behind by means of LEACH to craft the aggregator tree is to poise the power utilization in WSN. As LEACH pseudo-code selects the identical amount of the nodes as $\mathrm{CH}$, each $\mathrm{CH}$ assign the similar chip code towards their child nodes at that time the chip codes will be stable at each pace of the imitation.

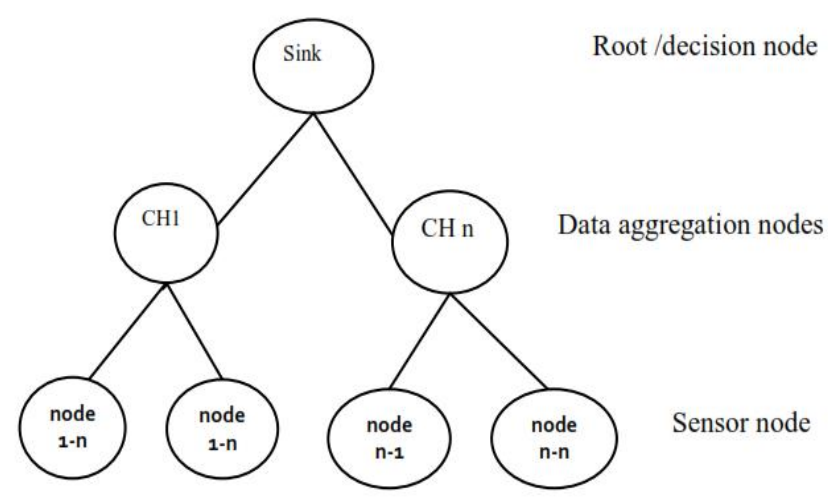

Figure 2. Tree Structure based on data aggregation 


\section{GSTEB}

General Self-Organized Tree-based Energy Balance routing protocol (GSTEB) shown in fig. 3 where network gathers information from time to time since a territory where every node frequently senses the location and throws the data return to Base station. The major goal of GSTEB is to attain a longer network lifetime for dissimilar applications. The simulation results show that when the data collected by sensor nodes is intensely correlative, GSTEB can modify the root and recreate the routing tree with little delay and small energy utilization. Thus a better balanced load is attained compared with the protocols such as LEACH, HEED, PEGASIS, PEDAP, and TBC. Because GSTEB is a self-organized protocol, it only consumes a little extent of energy in every round to change the topography for balancing the energy consumption. Entirely, leaf nodes can transmit data in the same TDMA time slot due to the short transmitting delay. GSTEB protract the lifetime by $100 \%$ to $300 \%$ [2] compared with PEGASIS. In some cases, the duration of the final node in the network is extra interested. A little minor transformation are made to build the performance of GSTEB similar with the purpose of PEDAP.

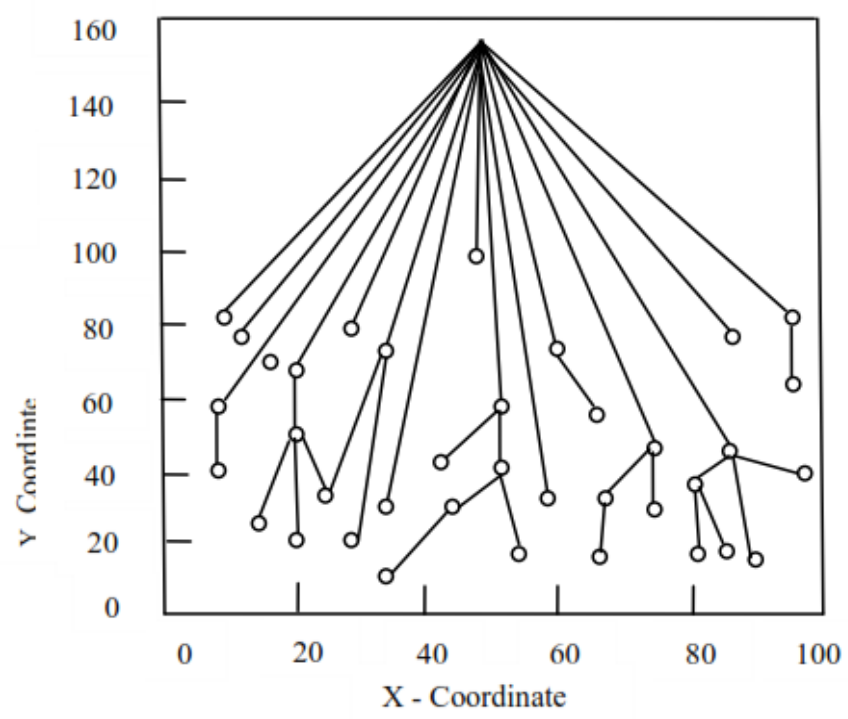

Figure 3. GSTEB Working

Tree-Based Clustering (TBC) is an improved protocol of LEACH. It forms several clusters in the same way as LEACH, and each cluster has a cluster-head $(\mathrm{CH})$. The nodes within a cluster construct a routing tree where the cluster-head is the root of it. For tree configuration, the cluster-head uses the distance information between the member nodes and itself. Each node is location-aware, it can estimate the distance between the root and itself. Every cluster is divided into some levels. The distance of a node to the root is the basis for determining its level in the cluster. The cluster-head is at level-0(root) and a node in level will choose the node in and nearest to itself as its parent node. Data transfer simultaneously happens between the nodes in two neighboring levels, and each node fuses the received data and transmits it to its parent. TBC is an excellent protocol in which each node records the information of its neighbors and builds topography through computing, which is similar to GSTEB. But some cluster-heads in the network consume more energy than other nodes when BS is located far away.

\section{RESEARCH MOTIVATION}

Wireless sensor nodes are deployed randomly and densely in a target region, especially where the physical environment is so harsh that the macro-sensor counterparts cannot be deployed. After deployment, the network cannot work properly unless there is sufficient battery power. In general, WSN may produce quite a substantial amount of data, so if data fusion could be used, the throughput could be reduced. Because sensor nodes are deployed densely, WSN might generate redundant data from multiple nodes, and the redundant data can be combined to reduce transmission. Many well-known protocols implement data fusion, but almost all of them assume that the length of the message transmitted by each relay node should be constant, i.e., each node transmits the same volume of data. General Self-Organized Tree-Based EnergyBalance routing protocol (GSTEB) builds a routing tree using a process where, for each round, BS assigns a root node and broadcasts this selection to all sensor nodes. Subsequently, each node selects its parent by considering only itself and its neighbors' information, thus making GSTEB a dynamic protocol.

\section{CONCLUSION}

In this paper, different tree -based clustering scheme for energy efficient data transfer in wireless sensor networks has been discussed. To form a tree in each cluster, the number of levels is decided according to the distance information of the member nodes. Through computer simulation in Matlab environment, an optimal number of levels in a cluster can be found. Based on this, a tree of nodes is formed in each cluster. As a result, it can minimize the energy consumption required for the communication between the nodes. In the future work, we will perform the simulation and will verify whether the scheme has significantly extended the network lifetime compared to the existing schemes or not. 


\section{REFERENCES}

[1] Y. Xiuwu, Fan Feisheng Zhou Lixing and Z. Feng, "WSN Monitoring Area Partition Clustering Routing Algorithm for Energy-Balanced," IEEE, pp. 80-84, 2016.

[2] S. Bera, S. Misra, Sanku Kumar Roy and Mohammad S. Obaidat, "Soft-WSN: Software-Defined WSN Management System for loT Applications," IEEE, pp. 1-8, 2016.

[3] N. A. M. Alduais, J. Abdullah, J. Abdullah, A. Jamil and L. Audah, "An Efficient Data Collection and Dissemination for IOT based WSN," IEEE, 2016.

[4] O. Singh, V. Rishiwal and M. Yadav, "Energy Trends of Routing Protocols for H-WSN," IEEE, 2016.

[5] R. Kumari and. P. Nand, "Performance Comparison of various Routing Protocols in WSN and WBAN," IEEE, pp. 427431, 2016.

[6] Hector Kaschel and ohanna Ortega, "Energy efficiency in routing protocols applied to WSN," IEEE, 2016.

[7] Asdianur Hadi and Ida Wahidah, "Delay Estimation using Compressive Sensing on WSN IEEE 802.15.4," IEEE, pp. 192-197, 2016.

[8] Mohd Zaki Shahabuddin, Halabi Hasbullah and Izzatdin A Aziz, "eliminary Framework of Topology Control Algorithm Achieve Node's Energy Efficiency," IEEE, pp. 259-263, 2016.

[9] Abhay kumar L. Gupta and Narendra Shekokar, "A Novel Approach to Improve Network Lifetime in WSN by Energy Efficient Packet Optimization," IEEE, 2016.

[10] B. Bengherbia, S. Chadli, M. Ould Zmirli and A. Toubal, "A MicroBlaze Based WSN Sink Node Using XBee Transceiver," IEEE, pp. 831-834, 2016.

[11] Gagandeep Kaur, Deepali and Rekha Kalra, "Improvement and Analys Security of WSN from Passive Attack," IEEE, pp. 4520-425, 2016.

[12] M. Wu, H. Liu and Q. Min, "Lifetime Enhancement by Cluster Head Evolutionary Energy Efficient Routing Model for WSN," IEEE, pp. 545-548, 2016.

[13] Roman Lara-Cueva, Rodolfo Gordillo, Liliana Valencia and Diego S. Ben, "Determining the Main CSMA Parameters for Adequate Performance of WSN for Real-time Volcano Monitoring System Applications," IEEE, pp. 1-9, 2016.

[14] Sanaa. S. Abd El dayem and M. R. M. Rizk, "An Efficient Authentication Protocol and Key Establishment in Dynamic WSN," IEEE, pp. 178-182, 2016. 